\title{
Alcances y limitaciones de Programas de Entrenamiento Cognitivo Computarizado Dirigidos al Trastorno por déficit de atención con hiperactividad
}

Scope and limitations of Computerized Cognitive Training Programs Directed to Attention Deficit Hyperactivity Disorder

\section{Escopo e limitações dos programas informatizados de treinamento cognitivo direcionados ao transtorno do déficit de atenção e hiperatividade}

María Lucia Blanco Parga, Jhon Erick Chacón Ruiz, Angie Tatiana Contreras Cifuentes, Maria Fernanda Corredor, Juliana Martínez Cárdenas, Ana Lorena Muñoz Valdés, Valeria Valentina Palencia Amaya, Luz Adriana Milena Penagos Fajardo, Sonia Gisela Ríos Cruz*, \& Maria Fernanda Zapata Orjuela Universidad Católica de Colombia

Citar este artículo así:

Blanco, M. L., Chacón, J. E., Contreras, A. T., Corredor, M. F., Martínez, J., Muñoz, A. L., Palencia, V.V., Penagos, L. A. M., Ríos, S. G.*, \& Zapata, M. F. (2020). Alcances y limitaciones de Programas de Entrenamiento Cognitivo Computarizado Dirigidos al Trastorno por déficit de atención con hiperactividad. Revista Enfoques, 3(1), 91-105. http://dx.doi.org/

Derechos de autor: Licencia Creative Commons AtribuciónNoComercial-SinDerivadas 4.0 internacional y 2.5 Colombia (CC BYNC-ND 2.5 (O)

Recibido: noviembre 14 de 2018

Revisado: mayo 23 de 2019 Aceptado: noviembre 26 de 2019

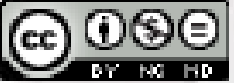

* Contacto para correspondencia: Sonía Ríos, email: sogiri3@gmail.com ORCID: https://orcid.org/oooo-0002-8632-2646 
Resumen | Antecedentes: en los últimos años se ha incrementado la utilización de programas de Entrenamiento Cognitivo Computarizado (ECC) como una alternativa terapéutica no invasiva, basados en la capacidad del cerebro para modificarse estructural y funcionalmente. Dirigidos a beneficiar tanto a la población sana como la diagnosticada con un trastorno específico, incluyendo allí el Trastorno por Déficit de Atención con Hiperactividad (TDAH). Sin embargo, su efectividad y la posibilidad de transferir los resultados a la vida cotidiana, aún es un tema controversial. De esta manera, el presente estudio tuvo como objetivo identificar los alcances y limitaciones de programas de ECC dirigidos a niños con TDAH. Método: Se tomó como referente artículos publicados en revistas científicas registradas en la base de datos PROQUEST, durante los años 2008 a 2018, bajo los comandos "Cognitive training", "ADHD", "children \& youth". Resultados: Se ubicaron 1229 artículos, en la búsqueda manual de la lista de referencia se identificaron 6 estudios que cumplieron con los requisitos de inclusión, es decir programas de ECC que fueron evaluados a través de estudios empíricos controlados, dirigidos a niños con TDAH. Conclusiones: La revisión permitió identificar que en general el ECC es útil para el tratamiento del TDAH en lo que respecta a las dificultades atencionales o de memoria de trabajo; es ambigua la evidencia en lo que respecta a su impacto en la vida cotidiana.

Palabras claves | TDAH, entrenamiento cognitivo, video juegos.

Abstract | Background: In recent years, the use of computerized cognitive training programs (CCT) has increased as a non-invasive therapeutic alternative, based on the brain's ability to modify itself structurally and functionally. The programs are aimed at the healthy population and at patients who have a specific disorder, including Attention Deficit Hyperactivity Disorder (ADHD). However, its effectiveness and the possibility of transferring the results to everyday life is still a controversial issue. The objective of this study was to identify the achievement and limitations of ECC programs aimed at children with ADHD. Method: Articles published in scientific journals registered in the PROQUEST database were taken as reference, during the years 2008 to 2018, under the commands "cognitive training", "ADHD", "children and youth". Results: 1229 articles were located, in the manual search of the reference list, 6 studies were identified that fulfilled the inclusion requirements. ECC programs were evaluated through controlled empirical studies, aimed at children with ADHD. Conclusions: The review made it possible to identify that, in general, the ECC is useful for the treatment of ADHD in terms of attentional difficulties or working memory. The evidence is ambiguous in terms of its impact on everyday life. 
Keywords $\mid$ ADHD, cognitive training, video game.

Resumo | Antecedentes: Nos últimos anos, o uso de programas computadorizados de treinamento cognitivo (TCC) aumentou como alternativa terapêutica não invasiva, com base na capacidade do cérebro de se modificar estrutural e funcionalmente. Os programas são direcionados à população saudável e a pacientes com um distúrbio específico, incluindo o Transtorno de Déficit de Atenção e Hiperatividade (TDAH). No entanto, sua eficácia e a possibilidade de transferir os resultados para a vida cotidiana ainda é uma questão controversa. $\mathrm{O}$ objetivo deste estudo foi identificar a conquista e as limitações dos programas de CEC voltados para crianças com TDAH. Método: Os artigos publicados em revistas científicas registradas na base de dados PROQUEST foram tomados como referência, nos anos de 2008 a 2018, sob os comandos "treinamento cognitivo", "TDAH", "crianças e jovens". Resultados: foram localizados 1229 artigos, na busca manual da lista de referências, foram identificados 6 estudos que preencheram os requisitos de inclusão. Os programas de CEC foram avaliados através de estudos empíricos controlados, voltados para crianças com TDAH. Conclusões: A revisão permitiu identificar que, em geral, o CEC é útil para o tratamento do TDAH em termos de dificuldades de atenção ou memória de trabalho. A evidência é ambígua em termos de seu impacto na vida cotidiana.

Palavras-chave |TDAH, treinamento cognitivo, videogame.

\section{Introducción}

El Déficit Atencional con Hiperactividad (TDAH) es un trastorno del neurodesarrollo que involucra factores genéticos y estructurales; caracterizados por un patrón de conductas de desatención, hiperactividad e impulsividad. Es una disfunción que afecta múltiples sistemas neuronales implicados en procesos cognitivos de orden superior, así como de tipo sensoriomotor; incluye el sustrato visual y el circuito prefrontal estriado (Cortese et al., 2012).

Las alteraciones a nivel comportamental del TDAH tienen mayor frecuencia e intensidad de lo que es habitual según la edad y el desarrollo del niño e interfieren de forma significativa tanto en el rendimiento escolar como en las actividades de la vida diaria (Willcutt, Doyle, Nigg, Faraone, \& Pennington, 2005). Los niños que son diagnosticados o presentan síntomas asociados al TDAH tienen mayor riesgo de presentar problemas académicos, dificultades en las relaciones familiares, además déficits en sus competencias sociales (Vélez-van-Meerbeke, Talero-Gutiérrez, Gonzalez-Reyes, \& Ibañez-Pinilla, 2008). 
De acuerdo con el informe de la Encuesta Nacional de Salud Mental (ENSM) (Ministerio de Salud, 2015), la prevalencia del TDAH a nivel mundial, registra una alta variabilidad entre el 1 al 20\%, adjudicada a la diversidad social y cultural, así como a condiciones de tipo metodológico; en Colombia una situación similar se reporta, por ejemplo, en niños de 7 a 11 años la prevalencia puede variar entre el 3,0 al 17,1 \%.

En el tratamiento del TDAH se han utilizado diversas estrategias terapéuticas, como el entrenamiento cognitivo (EC), cuyo propósito es aumentar la capacidad del niño para solucionar problemas y tomar decisiones más adecuadas, de tal forma que se aplican métodos tradicionales como ejercicios de lápiz y papel o tareas informatizadas (Merrill, 2007).

En los últimos años se ha diversificado los programas de EC que utilizan medios computarizados (ECC), ofertados para optimizar procesos de aprendizaje o mejorar funciones cerebrales, algunos dirigidos a poblaciones sanas como, el estudio realizado por $\mathrm{Yu}, \mathrm{Yu}, \mathrm{Fan}, \mathrm{y}$ Wang (2014) que tenía como objetivo analizar el impacto de un videojuego en el rendimiento de una clase de historia; en este se aplicó un diseño cuasiexperimental, se seleccionaron 103 alumnos (52 niños y 51 niñas), entre los 17 y 18 años, que cursaban segundo grado de secundaria superior. El grupo experimental fue instruido a través del programa denominado "The Age of Empires: The Conquerors" (CGBI), en una sesión de entrenamiento que duró seis horas en dos días, mientras el grupo control recibió inducción a través de una pizarra, tiza y un sistema de proyector multimedia. Los participantes del grupo experimental obtuvieron puntajes más altos que el control, con diferencias significativas.

Otros ECC han sido utilizado como sistemas de evaluación; tal como se evidencia en el estudio desarrollado por Hill et al. (2016) que tenían como objetivo presentar, describir y darle validez al método "Atención Visomotora" (VMA) para evaluar la atención sostenida y ejecutiva a través del rendimiento motor. Para ello ejecutaron cuatro experimentos en una plataforma computarizada; en cada experimento variaba la muestra, es decir, primero (adultos), segundo (jóvenes adultos comparados con niños de 7 a 12 años), tercero ( niños con trastornos del movimiento, comparados con desarrollo típico) y por último se incluyeron niños de los experimentos dos y tres; sin embargo, las condiciones experimentales eran las mismas, ya que los participantes en algunos ensayos debían realizar el seguimiento de un objeto (target) en una pantalla, a través de un lápiz óptico, mientras que en otros debían atender a cuatros objetos 
ubicados en cuadrantes diferentes de la pantalla que podían diferir en color, forma y una tercera condición combinada. Los autores encontraron una relación entre algunos resultados de la tarea VMA y los cuestionarios utilizados para evaluar la sintomatología asociada al TDAH.

También se destacan los ECC aplicados en procesos de habilitación o rehabilitación en trastorno específico, como TDAH (Aitana, 2016; Kraus \& Breznitz, 2009; Spencer-Smith \& Klingberg, 2015). Este tipo de estimulación ha demostrado tener efectos a nivel estructural, tal como lo reporta Hoekzema et al. (2010), puesto que identificaron actividad de la corteza orbito frontal izquierdo y del giro temporal medio (cuando se aplican ejercicios de control inhibitorio), así como incrementos en la actividad del cerebelo, área posterior derecha (al utilizar tareas de atención). En otro estudio se concluyó que producto de la estimulación se generaron cambios en el volumen de la materia gris (Hoekzema et al., 2011).

En general los estudios referidos a la efectividad funcional del ECC en el tratamiento del TDAH, así como su posible transferencia a la vida cotidiana, aún son limitados y controversiales; lo cual se hace evidente en algunas revisiones que se han hecho sobre el tema. Rivero et al. (2015) seleccionaron 14 estudios empíricos basados en vídeo juegos, dirigidos a estimular: la atención, memoria de trabajo y control inhibitorio; 10 juegos utilizaban el formato 3D, dos (2) eran simuladores que recreaban ambientes cotidianos y dos (2) eran minijuegos informatizados. De esta manera, los estudios incluidos en la revisión informan sobre mejoras en control inhibitorio, metacognición, planificación, memoria de trabajo e incluso habilidades académicas. No obstante, los autores reportan varias limitaciones de los estudios: dificultades en la selección del diseño dado que algunos no tienen grupo control, en otros no se evalúa la influencia de otras variables como la medicación o los niveles de dificultad del juego; además las muestras son pequeñas.

Orban, Rapport, Friedman, \& Kofler (2014) Ilevaron a cabo un metaanálisis, que tenía como objetivo entender los beneficios del EC en el TDAH mediante la aplicación de ejercicios informatizados; 17 estudios cumplieron con los requisitos de inclusión. De éstos se concluye que en general la efectividad de los programas de EC en el rendimiento académico o en los síntomas centrales del trastorno no están respaldados por evidencia empírica, falta correspondencia entre las funciones cognitivas que son estimuladas con el EC y sus resultados, en parte por el paradigma experimental que se usa en la evaluación, que algunas veces no 
corresponde con el proceso cognitivo entrenado. El objetivo del presente trabajo fue realizar una revisión de la evidencia disponible acerca de los programas de ECC dirigidos a niños con Trastorno por Déficit de Atención con Hiperactividad (TDAH) con el fin de identificar los alcances, limitaciones y posibles investigaciones que puedan desarrollarse.

\section{Método}

Los datos fueron tomados de artículos de investigación publicados en revistas científicas, incluidos en la base de datos Proquest (publicados en el periodo 2008 a 2018), se utilizaron como palabras claves: cognitive training y ADHA; bajo este comando se identificaron 7469 artículos, luego se seleccionaron los estudios dirigidos a niños y adolescentes quedando 1229 publicaciones. Por último, se realizó una búsqueda manual en la lista de referencia de los estudios recuperados, con el fin de identificar estudios empíricos de programas que aplicaran ECC a niños y adolescentes diagnosticados con TDAH; se descartaron intervenciones con neurofeedback, mindfulness, EC tradicional con lápiz y papel y revisiones sistemáticas o meta - análisis de tal forma que el análisis se realizó solo con 6 artículos.

\section{Resultados}

Los 6 artículos fueron divididos en dos grupos, en el primero se ubicaron los programas que replican paradigmas experimentales en el estudio de la cognición, o actividades de lápiz y papel empleadas en el EC tradicional, adaptadas a tareas informatizadas. Mientras que en el segundo el entrenamiento se realiza en un formato lúdico, con tareas que representan retos para los participantes, en la medida en que deben sortear una serie de obstáculos para conseguir un resultado (Davis, Bower, y Kollins, 2018). (Ver tabla 1) 
Tabla 1 | Características generales de los programas

\begin{tabular}{|c|c|c|c|c|c|}
\hline Autores & Proceso cognitivo & Participantes & Diseño & Programa & Resultados \\
\hline $\begin{array}{l}\text { Hovik, K. T., } \\
\text { Saunes, B. K., } \\
\text { Aarlien, A. K., } \\
\text { \& Egeland, J. } \\
(2013) \text {, }\end{array}$ & $\begin{array}{l}\text { Memoria de } \\
\text { trabajo }\end{array}$ & $\begin{array}{l}67 \text { niños } \\
10 \text { a } 12 \text { años }\end{array}$ & $\begin{array}{l}\text { Cuasiexperimental } \\
\text { con grupo } \\
\text { control activo, } \\
\text { seguimiento a los } \\
8 \text { meses }\end{array}$ & $\begin{array}{l}25 \text { días, } 8 \\
\text { ejercicios por día. } \\
\text { Duración } 30 \text { a } 40 \\
\text { minutos }\end{array}$ & $\begin{array}{l}\text { Mejoraron la } \\
\text { memoria de } \\
\text { trabajo }\end{array}$ \\
\hline $\begin{array}{l}\text { Lloyd, Brett, y } \\
\text { Wesnes (2010) }\end{array}$ & $\begin{array}{l}\text { Rendimiento } \\
\text { intelectual y } \\
\text { escolar }\end{array}$ & $\begin{array}{l}39 \text { niños } \\
9 \text { a } 13 \text { años }\end{array}$ & $\begin{array}{l}\text { Controlado } \\
\text { aleatorio con } \\
\text { grupo control } \\
\text { activo (figuras de } \\
\text { lego) }\end{array}$ & $\begin{array}{l}12 \text { semanas, } 6 \\
\text { semanas con } \\
\text { placebo activo y } \\
6 \text { semanas con } \\
\text { ejercicio. Además, } \\
\text { dos semanas antes } \\
\text { de la primera } \\
\text { evaluación de } \\
\text { línea base. }\end{array}$ & $\begin{array}{l}\text { Recuperación } \\
\text { de textos, } \\
\text { memoria } \\
\text { semántica } \\
\text { en recuerdo } \\
\text { libre como en } \\
\text { reconocimiento } \\
\text { y memoria } \\
\text { episódica }\end{array}$ \\
\hline $\begin{array}{l}\text { Egeland, } \\
\text { Aarlien y } \\
\text { Saunes. (2013) }\end{array}$ & $\begin{array}{l}\text { Dominios } \\
\text { neuropsicológicos, } \\
\text { el rendimiento } \\
\text { académico y el } \\
\text { funcionamiento } \\
\text { diario en el hogar } \\
\text { y la escuela }\end{array}$ & $\begin{array}{l}67 \text { niños } \\
10 \text { a } 12 \text { años }\end{array}$ & $\begin{array}{l}\text { Cuasiexperimental } \\
\text { con grupo control }\end{array}$ & $\begin{array}{l}13 \text { ejercicios } 5 \text { a } 7 \\
\text { semanas con una } \\
\text { duración de } 30 \text { a } \\
45 \text { minutos }\end{array}$ & $\begin{array}{l}\text { Mayor } \\
\text { rendimiento } \\
\text { en: memoria } \\
\text { de trabajo, } \\
\text { velocidad } \\
\text { psicomotora, } \\
\text { velocidad de } \\
\text { procesamiento, } \\
\text { lectura y } \\
\text { matemáticas. }\end{array}$ \\
\hline
\end{tabular}


Volumen 3 • Número 1 • enero - junio 2020 • Págs. 91 - 105 •

ISSN: 2389-8798 impreso / ISSN 2539-1445 (En línea) / Tunja, Boyacá (Colombia)

\begin{tabular}{|c|c|c|c|c|c|}
\hline Autores & Proceso cognitivo & Participantes & Diseño & Programa & Resultados \\
\hline $\begin{array}{l}\text { Davis et al. } \\
\text { (2018) }\end{array}$ & $\begin{array}{l}\text { Procesos } \\
\text { cognitivos } \\
\text { relacionados al } \\
\text { TDHA }\end{array}$ & $\begin{array}{l}80 \text { niños } \\
8 \text { a } 12 \text { años }\end{array}$ & $\begin{array}{l}\text { Cuasiexperimental } \\
\text { con grupo } \\
\text { control. El grupo } \\
\text { experimental } \\
\text { fue subdividido } \\
\text { en TDAH bajo y } \\
\text { moderado y el } \\
\text { grupo control sin } \\
\text { TDAH }\end{array}$ & $\begin{array}{l}\text { Software EVO } \\
\text { aplicado por los } \\
\text { padres } \\
27 \text { días, } 30 \text { a } 45 \\
\text { minutos, } 5 \text { veces } \\
\text { por semana }\end{array}$ & $\begin{array}{l}\text { Aumento en } \\
\text { los tiempos } \\
\text { de reacción, } \\
\text { mejoras en } \\
\text { la memoria } \\
\text { del trabajo } \\
\text { espacial y en el } \\
\text { procesamiento } \\
\text { visual, así como } \\
\text { disminución de } \\
\text { las respuestas } \\
\text { impulsivas }\end{array}$ \\
\hline $\begin{array}{l}\text { Shahmoradi, } \\
\text { Maghsoudi, } \\
\text { Najafi, \& } \\
\text { Pahlavanzadeh } \\
\text { (2017) }\end{array}$ & $\begin{array}{l}\text { Atención } \\
\text { focalizada y } \\
\text { sostenida }\end{array}$ & $\begin{array}{l}72 \text { niños } \\
7 \text { y } 12 \text { años }\end{array}$ & $\begin{array}{l}\text { Cuasiexperimental } \\
\text { con grupo control }\end{array}$ & $\begin{array}{l}\text { Software "space } \\
\text { face games } \\
\text { Una hora diaria } \\
\text { durante ocho } \\
\text { semanas, dos } \\
\text { veces por semana. } \\
\text { Total, de sesiones } \\
216\end{array}$ & $\begin{array}{l}\text { Rendimiento } \\
\text { en los procesos } \\
\text { atencionales } \\
\text { (disminución } \\
\text { en el número } \\
\text { de errores de } \\
\text { omisión. }\end{array}$ \\
\hline $\begin{array}{l}\text { Rosa, et al. } \\
\text { (2017) }\end{array}$ & $\begin{array}{l}\text { Memoria de } \\
\text { trabajo }\end{array}$ & $\begin{array}{l}\text { Seis }(6) \\
\text { pacientes } \\
\text { hospitalizados } \\
10 \text { a } 12 \text { años }\end{array}$ & $\begin{array}{l}\text { Ensayo clínico } \\
\text { aleatorio con } \\
\text { grupo control }\end{array}$ & $\begin{array}{l}\text { ACTIVATE } \\
\text { (4) cuatro } \\
\text { sesiones, cada una } \\
\text { con una duración } \\
\text { de } 45 \text { minutos, por } \\
12 \text { semanas }\end{array}$ & $\begin{array}{l}\text { Mejoras en } \\
\text { la memoria } \\
\text { de trabajo. } \\
\text { Respecto a } \\
\text { la memoria } \\
\text { visual no hubo } \\
\text { mejoras. }\end{array}$ \\
\hline
\end{tabular}




\section{ECC basado en tareas informatizadas}

Hovik, Saunes, Aarlien, \& Egeland. (2013), tenían como objetivo evaluar los efectos a largo plazo de un ECC dirigido a estimular la memoria de trabajo en niños con TDAH; para ello, seleccionaron una muestra de 67 niños, con edades comprendidas entre los 10 a los 12 años. Aplicaron un diseño con un pretest (una semana antes de la intervención), dos posts test (al finalizar el tratamiento y seguimiento ocho meses después) y grupo control. El grupo experimental participó durante 25 días en el programa de EC en la escuela, mientras que el grupo control recibía el tratamiento usual. El programa de ECC de memoria de trabajo, consistió en 13 diferentes ejercicios (tareas de letras, dígitos y ejercicios viso espaciales). El grupo de entrenamiento completó 10 de 15 ensayos de 8 ejercicios cada día, para un total de 115 ensayos por día; el tiempo estimado de entrenamiento era de 30 a 40 minutos. Se esperaba que hubiera un mejoramiento significativo en todas las medidas compuestas en el grupo de entrenamiento en comparación con el grupo control, predicción que fue confirmada, incluso los resultados se mantuvieron al menos ocho meses después. Los autores señalan como una de las limitaciones de su estudio el no contar con un grupo control normal con el cual comparar las ganancias de rendimiento.

Por su parte, Lloyd, Brett, y Wesnes (2010) Ilevaron a cabo un estudio controlado aleatorio, el cual tuvo como objetivo evaluar el efecto de un programa de enriquecimiento cognitivo basado en computadora, en el rendimiento intelectual y escolar de los niños; para ello seleccionaron una muestra de 38 niños diagnosticados con TDAH, que cursaban los grados 6,7 y 8 , su edad estuvo comprendida entre los 9 a 13 años. El ECC estaba estructurado en una serie de tareas que tenían como periférico dos botones, marcados como las palabras "no" y "sí". Además, la población se dividió en dos grupos; el primero es el grupo control activo el cual estuvo conformado por 22 estudiantes, con este grupo se evaluó la función cognitiva tres veces antes del inicio de la intervención (tiempo 1 o línea de base), nuevamente 6 semanas después de completar las sesiones de Lego (tiempo 2) y nuevamente 6 semanas después de completar el entrenamiento.

Por otro lado, el segundo grupo conformado por 14 participantes recibía el ECC. Los resultados de este estudio dan cuenta de la efectividad del ECC en tanto que los participantes presentaron mejoras significativas en varios aspectos del funcionamiento cognitivo, tales 
como recuperación de textos, memoria semántica (recuerdo libre como en reconocimiento) y memoria episódica. Entre otras limitaciones los autores consideran que se pudo presentar algunos sesgos en el diligenciamiento de uno de los instrumentos, como es el caso del "cuestionario de auto-reporte de comportamiento de los estudiantes", que fue diligenciado por los maestros y los alumnos, pero no hubo control de algunas variables que inciden en sus resultados, a saber, : la cultura, eventos cotidianos de la escuela o situaciones adversas de la vida de los niños que participaron en el estudio.

En la investigación realizada por Egeland, Aarlien, \& Saunes (2013), se analizó la transferencia del ECC en: otros dominios neuropsicológicos, el rendimiento académico y el funcionamiento diario en el hogar y la escuela. Para ello seleccionaron 67 niños, en edades entre 10 a 12 años, con un diagnóstico de TDAH. El tipo de investigación fue un ensayo controlado aleatorio (grupo control y experimental). Enste estudio se utilizó el "Cogmed's RoboMemo program", dirigido a estimular la memoria de trabajo; constó de 13 ejercicios que fueron aplicados en un ambiente controlado, por profesores o asistentes, que no participaron en las sesiones de evaluación. Las tareas sistematizadas eran seleccionadas de un algoritmo que aumenta o disminuye continuamente el nivel de dificultad de cada ejercicio según el rendimiento del niño. Incluía tres tareas de intervalo de letras (todas las secuencias hacia adelante), tareas de tres dígitos (una secuencia hacia adelante, dos secuencias hacia atrás) y siete tareas visuoespaciales en formato 2D o 3D (todas secuenciadas hacia adelante, bien sea estáticas o dinámicas). La aplicación se realizó de 5 a 7 semanas diarias con una duración entre 30 y 45 minutos. Los autores a partir de la evidencia concluyeron que posterior a la aplicación del programa hay mayor rendimiento en memoria de trabajo, velocidad psicomotora, velocidad de procesamiento, lectura y matemáticas.

\section{EEC basado en Videojuegos}

Davis et al. (2018), realizaron un estudio que tenía como objetivo evaluar la efectividad del software EVO desarrollado para tratar los procesos cognitivos relacionados al TDHA, el cual fue puesto a prueba en 80 participantes, 40 con TDHA y 40 sin este trastorno, que comprendían edades entre los 8 a 12 años. Los participantes pertenecientes al grupo que tenía TDAH fueron divididos en dos subgrupos, alto y moderado. Esta investigación manejó un diseño cuasi experimental longitudinal, que comprendió tres fases: sin intervención, con intervención y post intervención.

\section{$\boldsymbol{\epsilon} \mid 100$}


La fase sin intervención consistió en la administración del instrumento MINI-KID a los participantes para determinar si tenían TDAH y cuál era la gravedad de sus síntomas; después se les aplicó las pruebas CANTABAV y el TOVA, que son programas computarizados que ayudan a determinar cómo se realizan los procesos cognitivos en niños con TDAH y para evaluar cómo responden los niños a las tareas que involucran la atención e impulsividad; posteriormente, se les hizo una sección con el software EVO para hacer una serie de observaciones preliminares de cómo se comportaban los niños ante los juegos y finalmente, se les explicó a los papás en qué consistía el software para que ellos pudieran asesorar u orientar su uso en casa.

La fase de intervención se realizó en el hogar de cada participante durante 27 días, cada sesión se realizaba por espacio de 30 a 45 minutos diariamente, 5 veces por semana. Finalmente, la fase post intervención, es decir, el día 28 los investigadores analizaron los resultados obtenidos en aplicación del software EVO y volvieron a aplicar las pruebas CANTABAV y el TOVA; de manera simultánea los padres diligenciaron una serie de cuestionarios donde debían contestar cómo habían percibido y observado el rendimiento o comportamiento de su hijo. En el estudio se pudo determinar la efectividad del tratamiento, dado que se evidenció un aumento en los tiempos de reacción, mejoras en la memoria del trabajo espacial, en el procesamiento visual, así como disminución de las respuestas impulsivas.

La investigación realizada por Shahmoradi, Maghsoudi, Najafi, \& Pahlavanzadeh (2017), tenía como objetivo determinar el efecto de "Space face games" (un juego de identificar naves espaciales o extraterrestres en el espacio) sobre la atención (focalizada y sostenida) de los niños con TDAH. Para esto seleccionaron 72 niños entre 7 y 12 años, el muestreo se realizó por conveniencia y los niños fueron asignados al azar en dos grupos: uno experimental y otro de control. La herramienta de recolección de datos en este estudio fue la versión computarizada de la prueba de rendimiento continuo (CPT por sus siglas en inglés). El juego incluía un tablero, cuatro naves espaciales de color rojo, azul, amarillo y verde, además de muchas partes pequeñas como alienígenas con caras similares, de diferentes colores en las partes de sus caras, así como un reloj de arena.

Los sujetos tuvieron que mantener su atención por un tiempo en un estímulo relativamente simple, en este caso visual; cuando aparecía en el computador el estímulo señalado, debían responder presionando un botón. La prueba calificó cuatro índices de 
respuesta: errores de omisión, errores cometidos, tiempo de reacción y búsqueda correcta. Se realizaron sesiones de una hora bajo la supervisión de los investigadores durante 8 semanas, llevando a cabo dos sesiones por semana, para un total de 16 sesiones de intervención. Simultáneamente a las sesiones del grupo experimental, el grupo control tuvo sesiones con juegos como Lego y balones. El nivel de atención de ambos grupos se evaluó mediante el CPT antes y después de la intervención. Como resultados, los autores destacan el rendimiento en los procesos atencionales del grupo experimental después de 2 meses de práctica, reflejado en una disminución en el número de errores de omisión. La principal limitación del estudio fue el tiempo, debido a que no pudieron realizar un seguimiento para evaluar los efectos de la intervención a largo plazo, además tuvieron problemas de coordinación entre la asistencia de los niños a las sesiones de entrenamiento y sus clases.

Rosa et al. (2017) realizaron un estudio que tenía como objetivo evaluar a través de un ensayo clínico aleatorio con grupo control, la efectividad del programa de ECC denominado "Computerized Cognitive Remediation Training (CCRT) (ACTIVATE)" como tratamiento complementario al TDAH, allí seleccionaron seis (6) pacientes hospitalizados, cuyas edades oscilaban entre los 10 a 12 años. El programa se aplicó en cuatro sesiones, cada una con una duración de 45 minutos, durante un periodo de doce semanas. Las conclusiones a las cuales llegaron los investigadores desde la evidencia, es que el ECC podría conducir a mejorar la memoria de trabajo que solo la medicación, pero los pacientes que usaban medicación durante las sesiones se beneficiaron más de los programas de entrenamiento cognitivo. No se encontraron beneficios en la memoria visoespacial a corto plazo.

\section{Discusión}

El objetivo de este estudio era identificar los alcances y limitaciones del ECC dirigidos al TDAH. En general, las investigaciones donde se han aplicado o evaluado los ECC evidencian resultados favorables, la mayoría con efectos positivos en memoria de trabajo; así mismo se reporta, como una de las ventajas de este tipo de diseños computarizados, el aumento de la motivación de los participantes debido al formato lúdico y por la posibilidad de recibir una retroalimentación inmediata.

Respecto a las dificultades, los resultados son inconsistentes con respecto a la programación de las sesiones; por ejemplo, en algunos estudios se logran mejoras en los 
procesos cognitivos con 25 a 27 sesiones (Hovik, et al, 2013), mientras otros reportan resultados similares entre 13 a 17 (Egeland, et al, 2013). Un resultado similar fue reportado por Morrison y Chein. (2011), para lo cual sería importante utilizar diseños metodológicos que permitan identificar el impacto del número de sesiones en el rendimiento de las pruebas. Otro tópico para considerar es la utilización de los instrumentos de evaluación, dado que en algunos casos se limitan a valorar el proceso objetivo del entrenamiento y no exploran su impacto en otros.

A nivel metodológico se requiere aplicar diseños en los que se tenga un grupo control activo y otro pasivo, así mismo realizar seguimiento entre 6 a 8 meses después de terminar la aplicación del entrenamiento.

Es importante poder indagar el efecto de algunas variables tales como el acompañamiento u orientación de padres y maestros; en cuanto al formato lúdico utilizado no es claro qué puede ser más funcional, si las tareas informatizadas o el uso de videojuegos, puesto que en estos estudios los resultados fueron similares; de igual modo, es pertinente proponer y verificar la adecuación de las tareas de acuerdo con los intereses y habilidades de cada género.

Finalmente, en la medida en que surge este tipo de ECC se hace necesario la informatización de algunas pruebas, así como el uso de otro tipo de mediciones para evaluar el impacto, por ejemplo, al utilizar cuestionarios de tipo comportamental e incluso técnicas de neuroimagen.

\section{Referencias}

Cortese, S., Kelly, C., Chabernaud, C., Proal, E., Di Martino, A., Milham, M. P., \& Castellanos, F. X. (2012). Toward systems neuroscience of ADHD: A meta-analysis of $55 \mathrm{fMRI}$ sudies. American Journal of Psychiatry. https://doi.org/10.1176/appi.ajp.2012.11101521

Davis, N. O., Bower, J., \& Kollins, S. H. (2018). Proof-of-concept study of an at-home, engaging, digital intervention for pediatric ADHD. PLOS ONE. https://doi.org/10.1371/journal.pone.0189749

Egeland, J., Aarlien, A. K., \& Saunes, B. K. (2013). Few Effects of Far Transfer of Working Memory Training in ADHD: A Randomized Controlled Trial. PLoS ONE. https://doi.org/10.1371/journal.pone.0075660 
Hill, L. J. B., Coats, R. O., Mushtaq, F., Williams, J. H. G., Aucott, L. S., \& Mon-Williams, M. (2016). Moving to capture children's attention: Developing a methodology for measuring visuomotor attention. PLOS ONE. https://doi.org/10.1371/journal.pone.0159543

Hoekzema, E., Carmona, S., Ramos-Quiroga, J. A., Barba, E., Bielsa, A., Tremols, V., ... Vilarroya, O. (2011). Training-induced neuroanatomical plasticity in ADHD: A tensor-based morphometric study. Human Brain Mapping. https://doi.org/10.1002/hbm.21143

Hoekzema, E., Carmona, S., Tremols, V., Gispert, J. D., Guitart, M., Fauquet, J., ... Vilarroya, O. (2010). Enhanced neural activity in frontal and cerebellar circuits after cognitive training in children with attention-deficit/hyperactivity disorder. Human Brain Mapping. https://doi.org/10.1002/hbm.20988

Hovik, K. T., Saunes, B. K., Aarlien, A. K., \& Egeland, J. (2013). RCT of working memory training in ADHD: Long-term near-transfer effects. PLOS ONE. https://doi.org/10.1371/journal.pone.0080561

Kraus, T. H., \& Breznitz, Z. (2009). Can the error detection mechanism benefit from training the working memory? A comparison between dyslexics and controls - An ERP study. PLOS ONE. https://doi.org/10.1371/ journal.pone.0007141

Merrill, M. A. (2007). Captain's log: effectiveness of computerized cognitive training on ADHD symptoms. Tesis de doctorado: Sam Houston State University.

Ministerio de Salud y Protección Social. (2015). Encuesta Nacional de Salud Mental. http://www.visiondiweb. com/insight/lecturas/Encuesta_Nacional_de_Salud_Mental_Tomo_I.pdf

Morrison, A. B., and Chein, J. M. (2011). Does working memory training work? The promise and challenges of enhancing cognition by training working memory. Psycho. Bull., 18, 46-60. doi:10.3758/s13423-0100034-0

Lloyd, A.,Brett, D.,Wesnes, K. (2010).Coherence training in children with attention deficit hiperactivity disorder cognitive funtions and behavior changes. Alternative Therapies in Health and Medicine, 16, 4.

Orban, S. a., Rapport, M. D., Friedman, L. M., \& Kofler, M. J. (2014). Executive Function/Cognitive Training for Children with ADHD: Do Results Warrant the Hype and Cost? ADHD Report, 22(8), 8-14. https://doi. org/10.1521/adhd.2014.22.8.8 
Rosa, V. de O., Schmitz, M., Moreira-Maia, C. R., Wagner, F., Londero, I., Bassotto, C. de F., ... Rohde, L. A. P. (2017). Computerized cognitive training in children and adolescents with attention deficit/hyperactivity disorder as add-on treatment to stimulants: feasibility study and protocol description. 39(2), 65-76. doi:10.1590/2237-6089-2016-0039

Shahmoradi, Z., Maghsoudi, J., Najafi, M., \& Pahlavanzadeh, S. (2017). The effect of space face games on the amount of children attention with attention deficit hyperactivity disorders. Annals of Tropical Medicine and Public Health, 10(5), 1341. https://doi.org/10.4103/ATMPH.ATMPH_206_17

Spencer-Smith, M., \& Klingberg, T. (2015). Benefits of a working memory training program for inattention in daily life: A systematic review and meta-analysis. PLOS ONE, 10(3), 1-19. https://doi.org/10.1371/journal. pone.0119522

T.S., R., L.M.H., N., E.U., P., O.F.A., B., Strahler Rivero, T., Herrera Nunez, L. M., ... Amodeo Bueno, O. F. (2015). ADHD rehabilitation through video gaming: A systematic review using PRISMA guidelines of the current findings and the associated risk of bias. Frontiers in Psychiatry. https://doi.org/10.3389/fpsyt.2015.00151

Vélez-van-Meerbeke, A., Talero-Gutiérrez, C., Gonzalez-Reyes, R., \& Ibañez-Pinilla, M. (2008). Prevalencia del trastorno por déficit de atención con hiperactividad en estudiantes de escuelas de Bogotá, Colombia. Acta Neurológica Colombiana, 24(1), 6-12.

Willcutt, E. G., Doyle, A. E., Nigg, J. T., Faraone, S. V., \& Pennington, B. F. (2005). Validity of the executive function theory of attention-deficit/ hyperactivity disorder: A meta-analytic review. Biological Psychiatry. https://doi.org/10.1016/j.biopsych.2005.02.006

Yu, Z., Yu, W. H., Fan, X., \& Wang, X. (2014). An exploration of computer game-based instruction in the "world history" class in secondary education: A comparative study in China. PLOS ONE. https://doi.org/10.1371/ journal.pone.0096865 